\title{
The Study and Application of Grid Information Data Display Based on Web-SVG
}

\author{
WAN HuiJiang ${ }^{1, a}$, LIANG Ling ${ }^{2, b}$, MA JianWei, ${ }^{3, c}$, JIANG ZaiNeng $^{4, d}$ \\ 1,2,3,4 Guizhou Power Grid Co., Ltd., power dispatching control center, Guiyang, Guizhou 550002 \\ China \\ a408438504@qq.com; 'b361550717@qq.com ; ‘425054771@qq.com; d991980142@qq.com
}

Keywords: SVG; Web; database; correlation; AJAX

Abstract. This paper mainly introduced how to realize SVG display based on the Web Server, associated with graphics primitive and real-time data, obtained display of real-time data and historical data through Web server, made managers in other departments of the electric system share the data, hoping to make the correct command decision-making.

\section{Introduction}

In electric system, SCADA(Supervisory Control and Data Acquisition) is used to supervise the real-time operation situation of the grid, but SCADA system is the stand alone system in the grid system for the dispatching department. How to apply real-time data and historical data in each prefectural and municipal grid in SCADA system for other management except for the dispatching department through the Web Server transmission was the key in this study. SCADA refers to the process control and dispatching automatic system based on computers. SCADA can supervise and control operational equipment to realize data acquisition, equipment control, timing, measurement, time sequence recording, post disturbance review(PDR), statement generation, parameter regulation and alarm of various signals. The core technology is to realize uniform management and support variable structure, real-time and historical calculation of database. SCAD is composed of the remote terminal and control stations(system control centers). SCADA system is featured with distortionless scale, easy inquiry of characters, small modification of storage documents, embedded script language and supporting animation effects and interactive ability. SVG function based on XML is very powerful, so it is widely applied in many fields. In the electric system, SVG graph can display grid information data to display operational status of the grid.

\section{The Study on the Grid Information Data Display Based on Web-SVG}

\section{A. Web Server}

Different websites have different hardware demands of the Web server. If Web station is static, the hardware requirements of the Web server from high to low are shown as follows: network system, memory, disk system and CPU. If Web server mainly conducts the dense calculation, e.g. dynamic generation of Web page, the hardware demands for the server are successively shown as follows: memory, CPU, disk subsystem and network system. The demand situation of different applications on the key parts of the server is shown in Table 1.

Tab.1 Demand Distuation of Key Parts in the Server

\begin{tabular}{|c|c|c|}
\hline Application situations & CPU number & Memory number(MB) \\
\hline Only have static webspage & 1 & $128-256$ \\
\hline $\begin{array}{c}\text { Generation of dynamic } \\
\text { webspage }\end{array}$ & 2 & $256-1 \mathrm{~GB}$ \\
\hline LAN, 200times access/sec & 1 & $256-512$ \\
\hline LAN, 500 times access/sec & 2 & $512-1 \mathrm{~GB}$ \\
\hline LAN, 1000 times access/sec & Frebruary 4 & $1 \mathrm{~GB}-8 \mathrm{~GB}$ \\
\hline
\end{tabular}




\section{B. Design of the Database}

This system is to establish the data table to store the corresponding data information in Oracle10g database. Considering the data of each type in the system, the relational graph of data objects is shown in Figure 1.

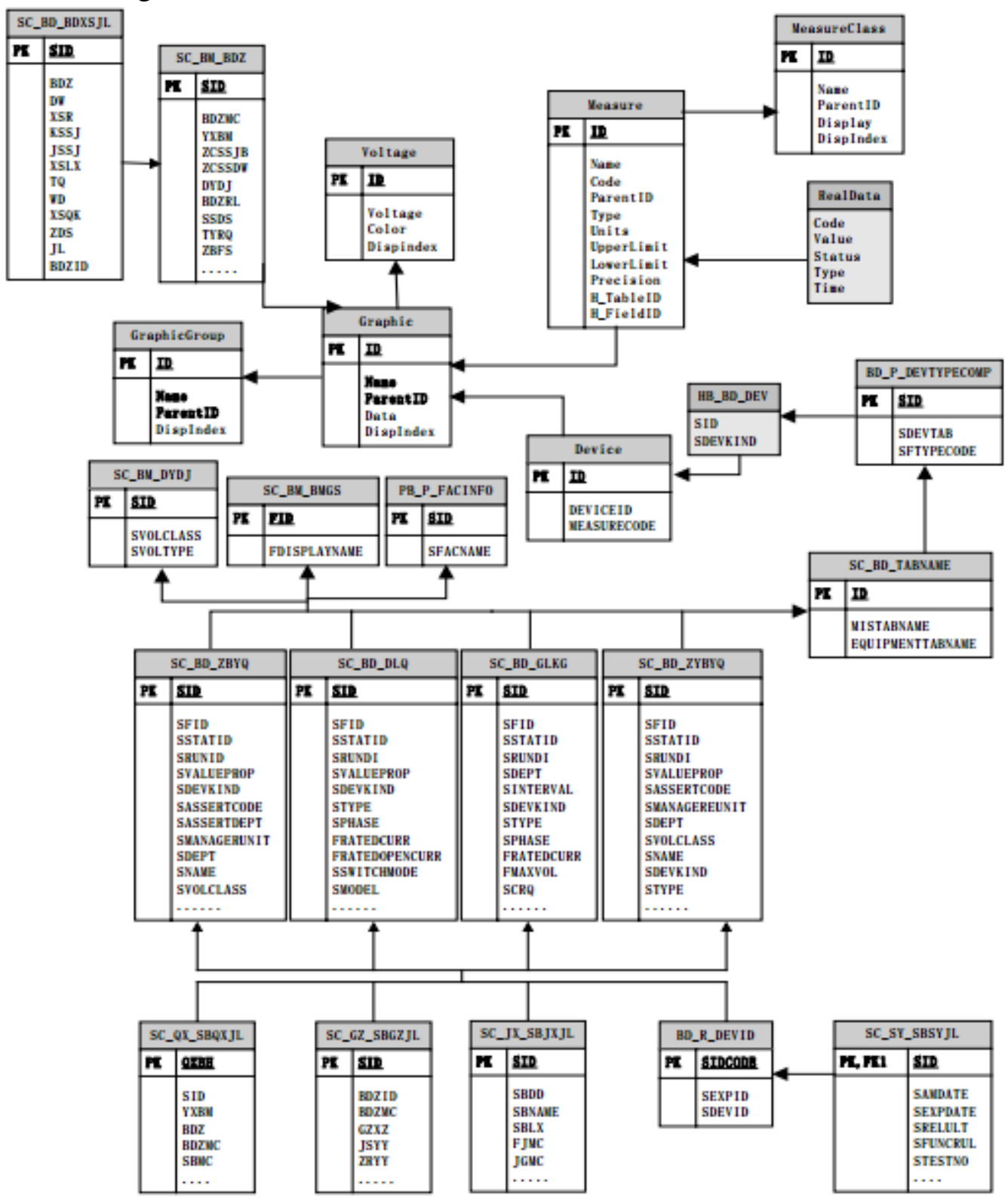

Fig.1 Relational Diagram of Data Objects

C. Real-time Display and Design of Curve Analysis System Data Table

In the primary connection diagram, SVG document is the two-bit diagram based on XML language to describe. In the data table, Blob type is used for storage. In the system, SVG graphic content is stored in Graphic table in Blob type. Graphic table stores some static quantity and belongs to the static table. Real-time data mean that dynamic quantity is stored in the dynamic table of real-time data table. The real-time data value in the real-time data table is associated with ID in the corresponding SVG content in DATA field of Graphic table. The expression form of the measuring point gets involved in "regional coding_factory station ID_equipment ID_classification standard”, such as "BD_210000031_220002367_KG”.

Tab.2 Real-time Data Table(Real Table)

\begin{tabular}{|c|l|l|}
\hline Name & Type & Notes \\
\hline Code & VARCHAR2(20) & Measuring point number \\
\hline Value & VARCHAR2(20) & Real-time data value \\
\hline Type & VARCHAR2(1) & Measuring point type \\
\hline
\end{tabular}




\begin{tabular}{|c|l|l|}
\hline Status & VARCHAR2(1) & State \\
\hline Time & VARCHAR2(20) & Data acquisition time \\
\hline
\end{tabular}

Table is the real-time data table structure figure. Real-time data table(Real Table) has 5 fields. Code corresponds to the measuring point number; value is the real-time data value; Status corresponds to the data status, Status=0(invalid), Status=1(valid), Status=-1(null value); Type corresponds to the measuring point type, Type $=0$ (analog quantity, shows telemeasuring data), Type $=1$ (switching value, only 0 and 1 , showing telecommand data); Time corresponds to the data acquisition time.

Tab.3 System Chart Series Table(Graphic Group Table)

\begin{tabular}{|c|c|c|c|c|}
\hline Name & Type & Allowable Null & Major key or not & Notes \\
\hline ID & NUMBER(10,0) & N & Yes & No \\
\hline NAME & VARCHAR2(20) & N & & $\begin{array}{c}\text { Graphic group } \\
\text { name }\end{array}$ \\
\hline ParentID & NUMBER(10,0) & N & & Father node ID \\
\hline DispIndex & NUMBER(10,0) & Y & & $\begin{array}{c}\text { Display position } \\
\text { index }\end{array}$ \\
\hline
\end{tabular}

Table 3 is the system graphic group table(Graphic Group Table) with the role of grouping all SVG documents in line with the prefectural and municipal areas and voltage, thus data division will be clearer. When users select certain voltage grade in a city, the system will conduct the automatic screening in line with this table to confirm the system graphic group selected by users.

Tab.4 System Graphic(Graphic Table)

\begin{tabular}{|c|c|c|c|c|}
\hline Name & Type & Allowable Null & Major key or not & Notes \\
\hline ID & NUMBER(10,0) & N & Yes & No \\
\hline NAME & VARCHAR2(20) & N & & $\begin{array}{c}\text { Graphic group } \\
\text { name }\end{array}$ \\
\hline ParentID & NUMBER(10,0) & N & & $\begin{array}{c}\text { System graphic } \\
\text { group ID }\end{array}$ \\
\hline Data & Blob & N & & $\begin{array}{c}\text { Dystem figure } \\
\text { index position }\end{array}$ \\
\hline DispIndex & NUMBER(10,0) & Y & & \\
\hline
\end{tabular}

Table 4 is the system graphic group table(Graphic Group Table) with the role of grouping all SVG documents in line with the prefectural and municipal areas and voltage, thus data division will be clearer. When users select certain voltage grade in a city, the system will conduct the automatic screening in line with this table to confirm the system graphic group selected by users.

Tab.5 System Graphic(Graphic Table)

\begin{tabular}{|c|c|c|c|c|}
\hline Name & Type & Allowable Null & Major key or not & Notes \\
\hline ID & NUMBER(10,0) & $\mathrm{N}$ & Yes & No \\
\hline NAME & VARCHAR2(20) & $\mathrm{N}$ & & $\begin{array}{l}\text { Graphic group } \\
\text { name }\end{array}$ \\
\hline ParentID & NUMBER(10,0) & $\mathrm{N}$ & & $\begin{array}{l}\text { System graphic } \\
\text { group ID }\end{array}$ \\
\hline Data & Blob & $\mathrm{N}$ & & System figure \\
\hline DispIndex & NUMBER(10,0) & $\mathrm{Y}$ & & $\begin{array}{c}\text { Display position } \\
\text { index }\end{array}$ \\
\hline
\end{tabular}

Table 5 is the system graphic(Graphic Table) with the role of associating all SVG figure through external key and system graphic group to store in DATA field through Blob type. SVG content stored in the DATA field is the most important data in the system diagram. In the system, majorities of functions focus on the core of SVG figure. The electric SVG uses BLOB to store in DATA field of system data table (Graphic Table). The storage format is XML format. The DATA field stores SVG contents. Table 6 is the voltage grade table(Voltage table). It is divided as different voltage grades with the main purpose of conducting color differentiation on elements of different voltage 
grades on the SVG figure. Each voltage grade corresponds to a color. The pale yellow stands for $500 \mathrm{KV}$, the purple is $220 \mathrm{KV}$, vermilion represents $110 \mathrm{KV}$, goldenrod is $35 \mathrm{KV}$, magenta color is $10 \mathrm{KV}$ and mazarine is $6 \mathrm{KV}$. DispIndex shows that position displayed on the browser.

Tab.6 Voltage Grade Table(Voltage Table)

\begin{tabular}{|c|c|c|c|c|}
\hline Name & Type & Allowable Null & $\begin{array}{c}\text { Major key } \\
\text { or not }\end{array}$ & Notes \\
\hline ID & NUMBER(10,0) & N & Yes & No \\
\hline Voltage & NUMBER(10,0) & Y & & Voltage grade value \\
\hline Color & VARCHAR2(20) & Y & $\begin{array}{c}\text { The corresponding color } \\
\text { of the voltage grade }\end{array}$ \\
\hline DispIndex & NUMBER(10,0) & Y & & Display position index \\
\hline
\end{tabular}

Table 7 is the measuring point classification table(MeasureClass) with the role of grouping the transformer substation in all measuring points, so that users can check relevant measuring point information. The measuring point classification table structure has 5 fields.

Tab.7 Measuring Point Classification Table(MeasureClass Tbale)

\begin{tabular}{|c|c|c|c|c|}
\hline Name & Types & Allowable Null & $\begin{array}{c}\text { Major key } \\
\text { or not }\end{array}$ & Notes \\
\hline ID & NUMBER(10,0) & N & Yes & No \\
\hline NAME & VARCHAR2(20) & Y & & $\begin{array}{c}\text { Measuring point } \\
\text { classification name }\end{array}$ \\
\hline ParentID & NUMBER(10,0) & Y & & $\begin{array}{c}\text { Father node ID } \\
\text { Display }\end{array}$ VARCHAR2(20) \\
\hline DispIndex & NUMBEring point \\
\hline
\end{tabular}

\section{The Application of Grid Information Data Display Based on Web-SVG}

Real-time data and historical data display platform has the main function is based on SVG to show it to users with primary connection diagram, real-time dispatching information, dispatching information historical state in the simple and intuitive manner. Meanwhile, it also allows users to amplify and scale SVG figure, amplify and scale mouse pulley, amplify frame and wander, so that users can achieve these functions through the simple operations. These pictures are distortionless and can greatly meet user demands. In the use process, users can gain the primary connection diagram, while the system executes the corresponding function and write real-time data in the corresponding measuring point position of the primary connection diagram and display it, ensuring to update data at $5 \mathrm{~s}$ of interval(According to user demands, it can be improved to $2 \mathrm{~s}$ at the soonest), so as to ensure that users can timely observe update changes of real-time data. The dispatching data is shown in Figure 2 in real time.

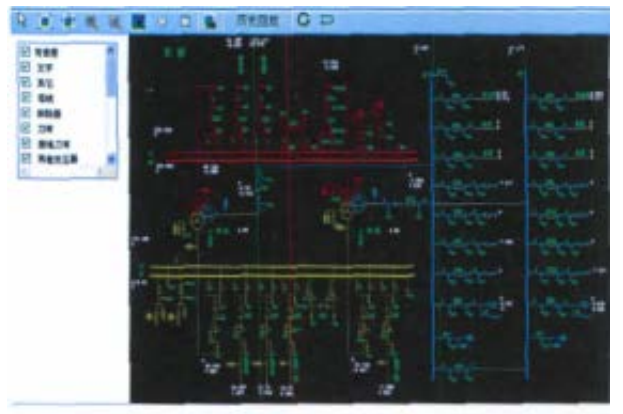

Fig. 2 The Real-time display of dispatching data

The relevant data to be measured in the figure are dynamically changed to embody operational status of the grid. Users can inquire historical data as required. This platform can display voltage, current, active power, inactive power, phase angle and temperature in the grid, so as to provide the reliable and effective data support for user command production in the grid management. 


\section{Conclusions}

It is proven that SVG graphics can be used as the display platform of real-time data and historical data in the grid. The graphic is simple, excellent, has no flickering and shows dynamic effects, thus users can clearly and systematically observe changing tendency of measuring point in the grid and have the clear understanding on the operation state in the grid.

\section{References}

[1] Cao Junwei, Yuan Zhongda, Ming Yangyang and Zhang Huaying, the Summary of Energy Internet Big Data Analysis Technology[J], South Grid Technology, 2015, 11: 1-12;

[2] Wang Quanqiang, Liu Min and Chengli, the Study on the Electric Big Data Technology in the Smart Grid[J], Electronic Technology and Software Engineering, 2016, 01: 181-182;

[3] Zhang Jianye, Liu Kui, Zhang Jianguang, Liu Yongting and Zhou Yuelin, the Study and Realization on the Big Data Visualization Technology in the Grid[J], Science Technology Innovation, 2017, 29: 5-6;

[4] Qu Chaoyang, Xiong Zeyu,Yan Jia, Xin Peng and Qu Nan, the Visualized Scene Management Method of Electric Big Data 3D Panorama Based on Space Division[J], Journal of North China Electric Power University(Natural Science Version), 2016, 02: 23-29;

[5] Zhang Rui, Liu Daowei, Chen Shuyong, Ma Shiying, Deng Chunyu, Shi Mengjie and Wu Qian, the Visualization Design of the Big Grid Panorama Safety Defense System of Information Driving[J], Electronic Information and Communication Technology, 2016, 12: 46-51 